\title{
Simultaneous Characterization of Lipid sn-Positional and Double-Bond Positional Isomers using On-Demand Electrochemical Mass Spectrometry
}

\author{
Shuli Tang, ${ }^{a}$ Xin Yan *a \\ [a] Department of Chemistry, Texas A\&M University, 580 Ross St., College Station, TX 77845
}

\begin{abstract}
In this work, we present an on-demand electrochemical mass spectrometry workflow for lipid structural characterization with the capability of identifying double-bond and $s n$-positional isomers. Two voltage-controlled electrochemical reactions, interfacial electro-epoxidation and anodic corrosion of a cobalt electrode, are cascaded in this strategy. The epoxidized products and Co-adducted ions of lipids are fragmented in tandem MS to generate structure characteristic fragments that indicate double-bond and $s n$-positions. The unique feature of this workflow lies in the sequential localization of double bonds, elucidation of sn-positions, and collection of native lipid information in a single system and simply by tuning ESI voltages. This feature, as well as low sample consumption, no need for extra apparatus, and quantitative analysis, should allow wide applications in the lipidomic field.
\end{abstract}

\section{Introduction}

Lipids are principal components of biological membranes, and play vital roles in maintaining cellular functions. ${ }^{1,2}$ Recent studies have recognized the dysregulated lipid metabolism in the pathogenesis of many diseases including obesity, ${ }^{3}$ diabetes, ${ }^{4}$ cancers, ${ }^{5}$ cardiovascular diseases, ${ }^{6}$ and neurodegenerative disorders. ${ }^{7}$ Full characterization of lipid compositions is significant for identifying lipid networks and understanding disease mechanisms. ${ }^{8-11}$ Complete lipid structural elucidation has proven to be quite difficult since lipids are structurally diverse and often contain various types of isomers. ${ }^{12}$ As the major components of eukaryotic membranes and significant molecules in cell signaling, ${ }^{2}$ glycerophospholipids (GPLs) are found to have numerous structural isomers due to the variations in their head-groups, fatty acyl chain lengths, degree of unsaturation, sn-positions (relative positions of fatty acyls esterified on the glycerol backbone), as well as the positions and configurations of double bonds. ${ }^{13}$ Such small variations in structures could result in unique roles in lipid homeostasis and pathology, and can be used as biomarkers in disease diagnosis. ${ }^{14-16}$ For example, Ellis et. al. demonstrated that changes in relative concentrations of lipid double-bond and $s n$-positional isomers reflect cell-specific enzyme activities. ${ }^{17}$ Cao and co-workers reported that subtypes of breast cancer cells display different compositions of double-bond and sn-positional isomer, and human lung cancerous tissues can only be distinguished from normal tissues using both kinds of isomers. ${ }^{18}$ These results highlight the importance of structure characterization of lipids at the isomer level in disease diagnosis and in understanding their pathologies. 
Mass spectrometry (MS) coupled with tandem MS (MS/MS) has been widely used in lipid analysis. ${ }^{19-21}$ Tandem MS in the form of collision-induced dissociation (CID) aids the lipid assignments of class and chain length isomers from the fragment ions associated with headgroup loss and fatty acyl chain loss. To achieve lipid structure characterization at the double-bond and sn-positional isomer level, recent efforts have gone into developing new derivatization and ion activation methods. Chemical derivatization methods such as methoxylation, ${ }^{22,}{ }^{23}$ methylthiolation, ${ }^{24-26}$ olefin cross-metathesis, ${ }^{27}$ ozonolysis, ${ }^{17,}{ }^{28-30}$ Paternò-Büchi (PB) reaction, ${ }^{31-34}$ as well as the plasma-induced ${ }^{35-37}$ and off-line meta-chloroperoxybenzoic acid (m-CPBA) epoxidation ${ }^{38,39}$ have been developed to functionalize lipid double-bonds and to produce characteristic fragment ions in tandem MS to locate double-bond positions. Novel ion activation methods including ultraviolet photo-dissociation (UVPD), ${ }^{40}$ ozone-induced dissociation (OzID), ${ }^{41}$ and electron impact excitation of ions from organics (EIEIO) ${ }^{42}$ also successfully achieved double-bond localization. Moreover, UVPD, OzID, EIEIO and radical induced fragmentation are able to identify snposition of lipid isomers. ${ }^{13,43-46}$ These studies build the field of structural lipidomics and shed light on lipid biochemistry.

Our previous work described the first electrochemical $(E C)$ probe for lipid structural analysis, and showed that it allows the elucidation of double-bond positional isomers. ${ }^{47}$ This was achieved by developing a novel voltagecontrolled electro-epoxidation reaction at the Taylor cone interface using a wire-in-a-capillary ESI emitter with a large orifice (LO-ESI emitter), followed by CID fragmentation of the epoxides to form two characteristic ions in MS/MS. The electro-epoxidation of double-bonds can be rapidly switched on/off by simply changing the applied electrospray voltage.

Encouraged by these powerful and on-demand features, we develop a novel EC derivatization method for characterization of lipid sn-positional isomers in this work. We found that cobalt ions $\left(\mathrm{Co}^{2+}\right)$ produced by anodic corrosion of ESI electrode can be used to form complexes binding to lipids at the sn-positions, which leads to characteristic fragment ions in CID. It is worth noting that the formation of Co-lipid complexes in this method is voltage-dependent, which allows us to cascade it with electro-epoxidation of lipids for simultaneous identification of double-bond isomers. Therefore, we present an EC-MS-based workflow where double-bond location, sn-position as well as native lipid structure can be sequentially determined by tuning ESI voltages. The characterization can be completed in a single sample.

\section{Results and Discussion}

\section{EC-MS-based Workflow for Simultaneous Characterization of Lipid sn-Positional and Double-Bond Positional Isomers}

The on-demand EC-MS-based workflow is illustrated using a pair of unsaturated sn-positional lipid isomers, phosphatidylcholine (PC) 18:1 (9)-16:0 and PC 16:0-18:1 (9). We dissolved the two PC lipids in a mixture of solvent (acetonitrile/water $=1 / 4, \mathrm{v} / \mathrm{v}$ ) and $10 \mathrm{mM}$ hydrochloride acid to achieve a concentration of $20 \mu \mathrm{M}$. Samples were 
loaded into two LO-ESI emitters separately, then placed $8 \mathrm{~mm}$ away from the inlet of a mass spectrometer (Thermo LTQ Orbitrap Velos Pro). A Co wire was inserted into the LO-ESI emitter to serve as the ESI electrode.

(a) Schematic of the set-up of LO-ESI-MS

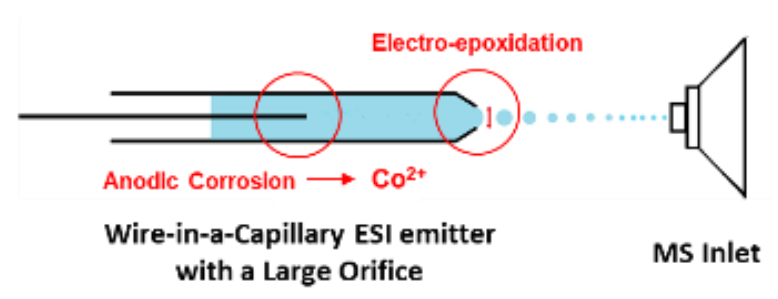

(b) selected ion chronograms

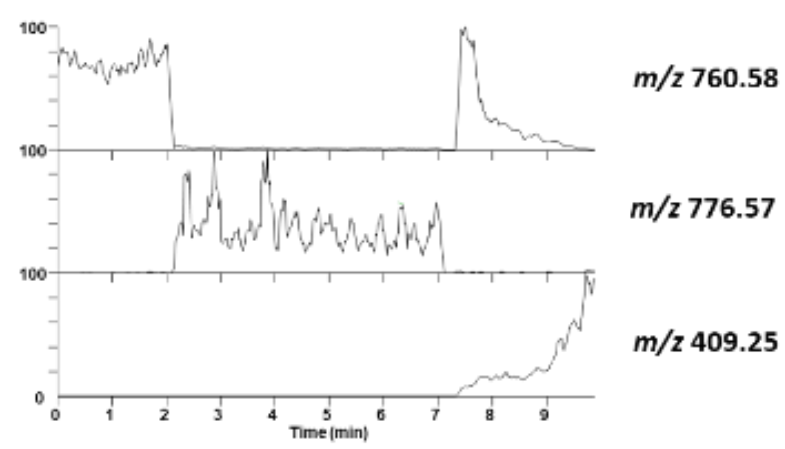

(c) Pronated PC 18:1 (9)-16:0

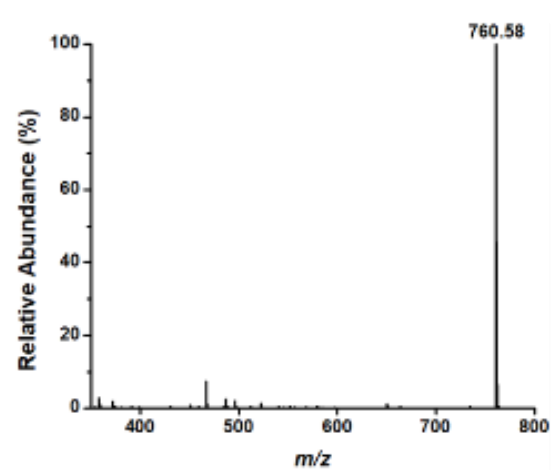

(d) Epoxidized PC 18:1 (9)-16:0

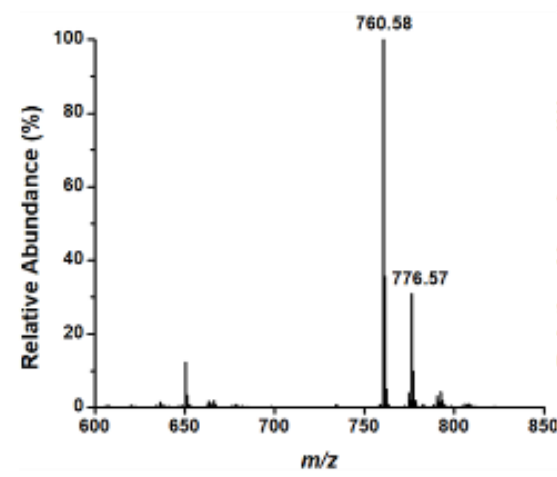

(e) Co-Adduced PC 18:1 (9)-16:0

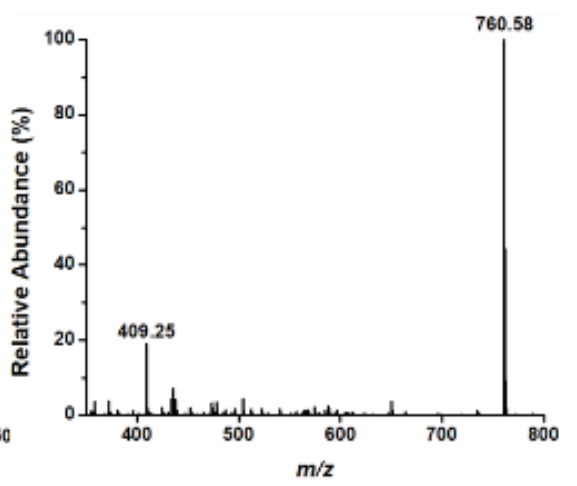

Figure 1. (a) Schematic of the set-up of LO-ESI used in the on-demand EC-MS-based workflow, and (b) selective ion chronograms of the protonated native lipid PC 18:1(9)-16:0 at $\mathrm{m} / \mathrm{z}$ 760.58, the product ions of electro-epoxidation of double bonds at $\mathrm{m} / \mathrm{z} 776.57$ and the Coadducted lipid ions at $m / z$ 409.25. Mass spectra of (c) protonated, (d) epoxidized, and (e) Co-adducted PC 18:1(9)-16:0.

The workflow achieves (i) localization of double bonds by fragmenting the product of electro-epoxidation of lipids when the voltage is $1.8 \mathrm{kV}$; (ii) elucidation of $s n$-positions by fragmenting the lipid-Co complex ions when the voltage is switched to $3.0 \mathrm{kV}$. (iii) Interestingly, one can also obtain the MS spectrum of native lipids at a voltage of $3.0 \mathrm{kV}$ with the Co electrode positioned in the middle of the LO-ESI emitter.

We now describe the characterization of isomeric PC 18:1_16:0 using the on-demand EC-MS-based workflow of a single sample injection. Protonated PC 18:1 (9)-16:0 and PC 16:0-18:1 (9) were both observed at $m / z 760.5760$. CID fragmentation of the protonated lipids produced the ions associated with headgroup loss and fatty acyl chain loss which allow identification of the backbone of lipids. For example, the tandem spectrum of protonated PC 18:1 (9)-16:0 shows characteristic ions at $\mathrm{m} / \mathrm{z} 577.50, \mathrm{~m} / \mathrm{z} 522.34$ and $\mathrm{m} / \mathrm{z} 496.25$ (Figure 3 a), corresponding to the structures 2, 3, and 4 in Figure 2. The epoxidized PC 18:1 (9)-16:0 and PC 16:0-18:1 (9) were found at $m / z$ 776.5720. Fragmentation of the mono-epoxidized products results in the cleavage of the epoxide ring at both sides of $\mathrm{C}-\mathrm{O}$ bond, generating two diagnostic ions that have 15.9954 Da mass difference to pinpoint double-bond positions. The presence of ions at $m / z$ 634. 4402 and $m / z 650.4351$ in tandem spectrum of epoxidized PC 18:1 (9)-16:0 (Figure 3b) 
confirms that the double-bond was located between the C-9 and C-10 in its unsaturated fatty acyl chain (FA) 18:1. Figure 2 (b) provides the fragmentation pathway of epoxidized PC 18:1 (9)-16:0 and the corresponding structures of the two diagnostic ions. Co-adducted PC 18:1 (9)-16:0 and PC 16:0-18:1 (9) ions were found at $m / z$ 409.2543. snPositions in lipids are then identified via tandem spectra of the Co-adducted products. PC 18:1 (9)-16:0 has its FA 18:1 (9) at $s n-1$ position and FA 16:0 at $s n-2$ position (structure 1 in Figure 2). When the $\mathrm{Co}^{2+}$ ions are bound to PC 18:1 (9)-16:0, it is proposed that a five-membered ring prefers to form containing the phosphate group and FA 16:0 at sn-2 position (structure $\mathbf{8}$ in Figure 2), and then this gives a pair of fragment ions associated with the FAs attached to $s n-2$ position (see pathway a in Figure $2 c$ ). Alternatively, an analogous fragmentation pathway that occurs via a six-membered ring (see pathway $\mathbf{b}$ in Figure 2) may also exist in a small amount. The tandem spectrum of Coadducted PC 18:1 (9)-16:0 ions contains two pairs of diagnostic ions having significantly different intensities (Figure 3c). The dominant fragment ions have $m / z$ at 314.1629 and 504.3413, corresponding to structure 9 and 10 in Figure 2. Ions at $m / z 340.1782$ and 478.3259 are observed in low abundance, and these correspond to structure $\mathbf{1 2}$ and 13 (Figure 2). In contrast, dominant species at $\mathrm{m} / \mathrm{z} 340.1782$ and 478.3259 are observed in the fragmentation of Co-adducted PC 16:0-18:1 (9) ions (Figure 3d).

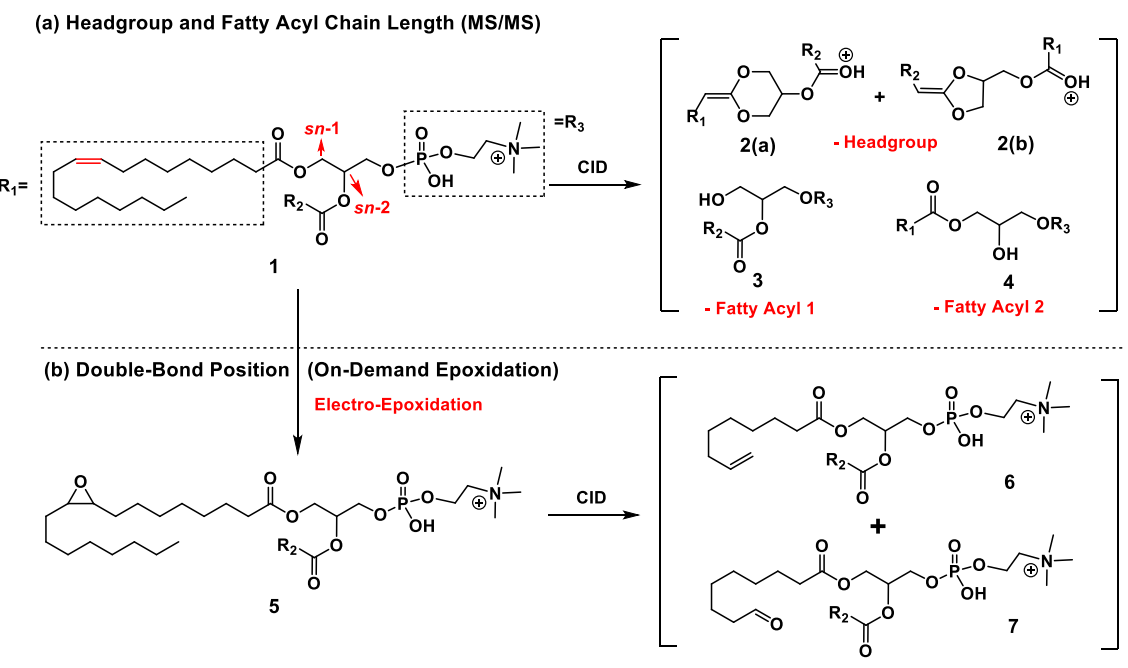

(c) sn-Position (On-Demand Electrolysis)

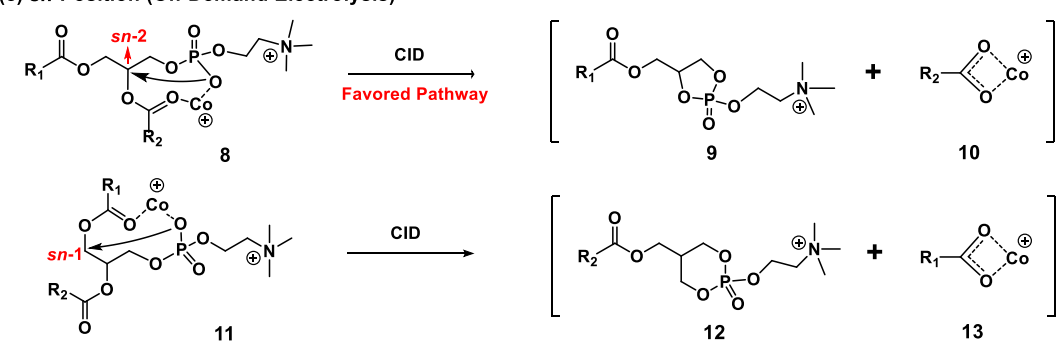

Figure 2. Structure characterization of PC 18:1 (9)-16:0 using the on-demand EC-MS-based workflow $\left(\mathrm{R}_{2}=n-\mathrm{C}_{15} \mathrm{H}_{31}\right)$. 

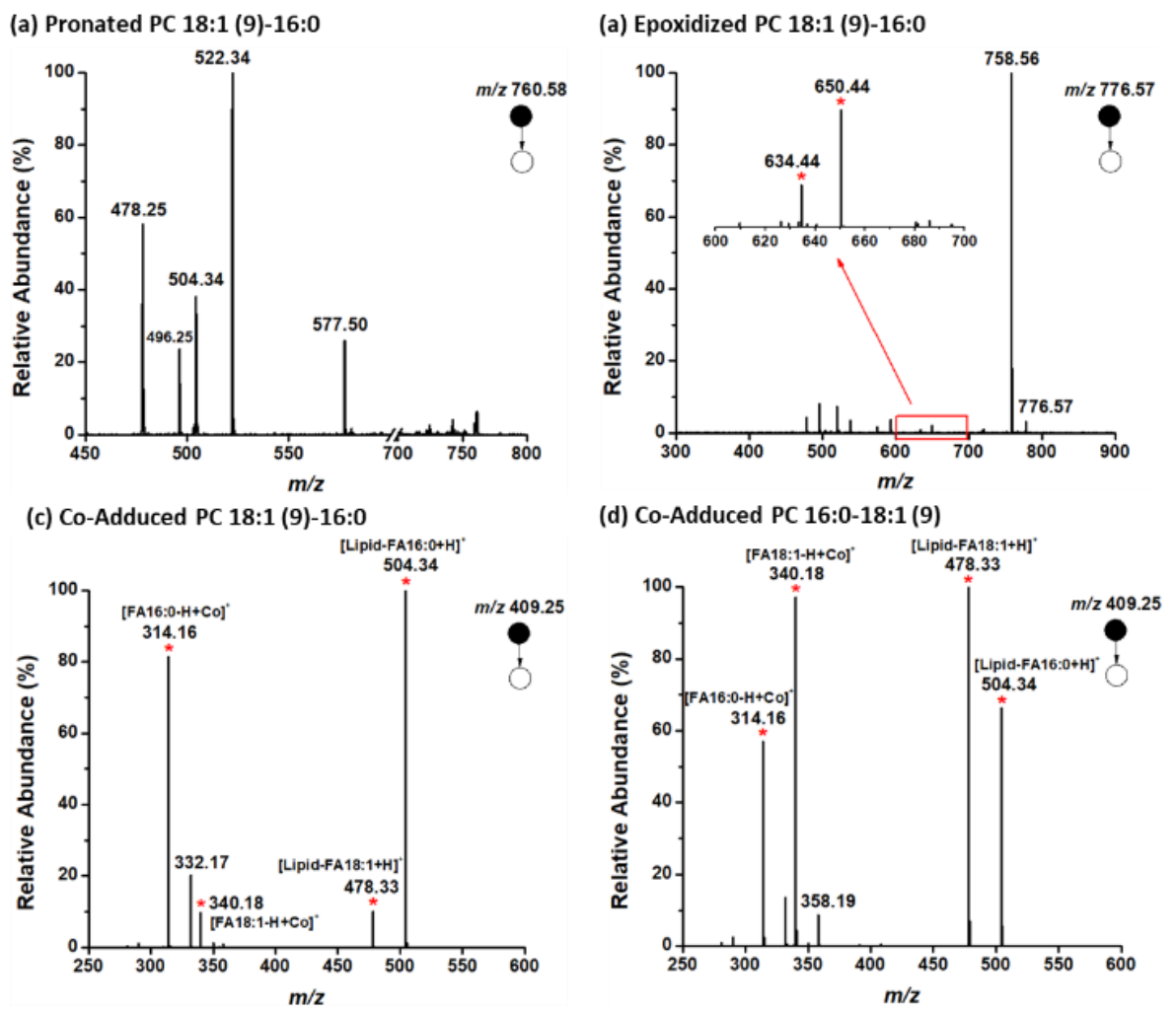

Figure 3. Tandem mass spectra of (a) protonated PC 18:1 (9)-16:0; (b) electro-epoxidized PC 18:1 (9)-16:0; (c) Co-adducted protonated PC 18:1 (9)-16:0, and (d) Co-adducted protonated PC 16:0-18:1 (9).

\section{Quantitation of Lipid Double-Bond and sn-Positional Isomers}

Quantitation of lipid double-bond and sn-positional isomers can be achieved based on diagnostic ions intensities. A pair of lipid double-bond positional isomers, PC 18:1 (6)-18:1 (6) and PC 18:1 (9)-18:1 (9), and two lipid snpositional isomers, PC 18:1 (9)-16:0 and PC 16:0-18:1 (9) are selected for the quantitation experiments.

In the quantitative analysis of double-bond positional isomers, a series of mixed solutions of PC 18:1 (6)-18:1 (6) and PC 18:1 (9)-18:1 (9) were used, with the concentration of PC 18:1 (6)-18:1 (6) being constant at $30 \mu \mathrm{M}$ and PC 18:1 (9)-18:1 (9) varying from $10 \mu \mathrm{M}$ to $50 \mu \mathrm{M}$. The two isomers were electro-epoxidized upon the application of a $1.8 \mathrm{kV}$ voltage in a LO-ESI emitter and they generated both mono-epoxidized and di-epoxidized products at $\mathrm{m} / \mathrm{z}$ 802.5996 and $m / z$ 818.5936, respectively (see Supporting Information S4 for details). The mono-epoxides were then mass-selected and fragmented by CID. In their tandem mass spectra, PC 18:1 (6)-18:1 (6) showed diagnostic ions at $\mathrm{m} / \mathrm{z} 618.4158$ and 634.4122 , and the ions at $\mathrm{m} / \mathrm{z} 660.4618$ and 676.4572 were seen as the diagnostic ions of PC 18:1 (9)-18:1 (9) (Figure 4a and b). The total ion intensities ratios of the two pairs of double-bond diagnostic ions, e.g. total ion intensities of $\mathrm{m} / \mathrm{z}$ at 660.4618 and 676.4572 from PC $\Delta 9$ divided by the intensities of $\mathrm{m} / \mathrm{z} 618.4158$ and 634.4122 from PC $\Delta 6$, were plotted against their concentration ratios. Good linearity $\left(R^{2}=0.9967\right)$ was obtained (Figure 4c). 
To quantify the sn-positional isomers, a series of mixtures of PC 18:1 (9)-16:0 and PC 16:0-18:1 (9) was analyzed, with the total concentration kept constant at $50 \mu \mathrm{M}$, and molar ratios varied. In the tandem mass spectrum of Coadducted PC 18:1 (9)-16:0, the ratio calculated by using the total intensities of ions at $m / z 314.1629$ and 504.3413 divided by the total ion intensities of the four diagnostic fragment ions at $\mathrm{m} / \mathrm{z} 314.1629, \mathrm{~m} / \mathrm{z} 504.3413, \mathrm{~m} / \mathrm{z}$ 340.1782 and $m / z 478.3259$ is 0.8622 , while the calculated ratio for Co-adducted PC 16:0-18:1 (9) is 0.2649. A good linear relationship was found when the ratios were plotted against the molar percentage of PC 18:1 (9)-16:0 in the mixed solutions $\left(R^{2}=0.9965\right)$ (Figure $\left.4 d\right)$. These results demonstrate that the on-demand EC-MS-based workflow can be used for quantification of both lipid double-bond and $s n$-positional isomers.

The detection limit was evaluated using PC 18:1 (9)-16:0 and PC 16:0-18:1 (9). The double-bond diagnostic ions can be observed with acceptable signal/noise at $50 \mathrm{nM}$ for PC 18:1 (9)-16:0 and $10 \mathrm{nM} \mathrm{PC} \mathrm{16:0-18:1} \mathrm{(9),} \mathrm{which} \mathrm{is}$ comparable to the value reported in our previous work (10 nM for PC 18:1 (9)-18:1 (9) and 80 nM PC 18:1 (6)-18:1 $(6))^{25}$. The $s n$-position diagnostic ions can be clearly seen, and used to identify sn-positions of the two lipid isomers even at $10 \mathrm{nM}$ (see Supporting Information S5 for details).

(a) Epoxidized PC 18:1 (9)-18:1 (9)

(b) Epoxidized PC 18:1(6)-18:1(6)
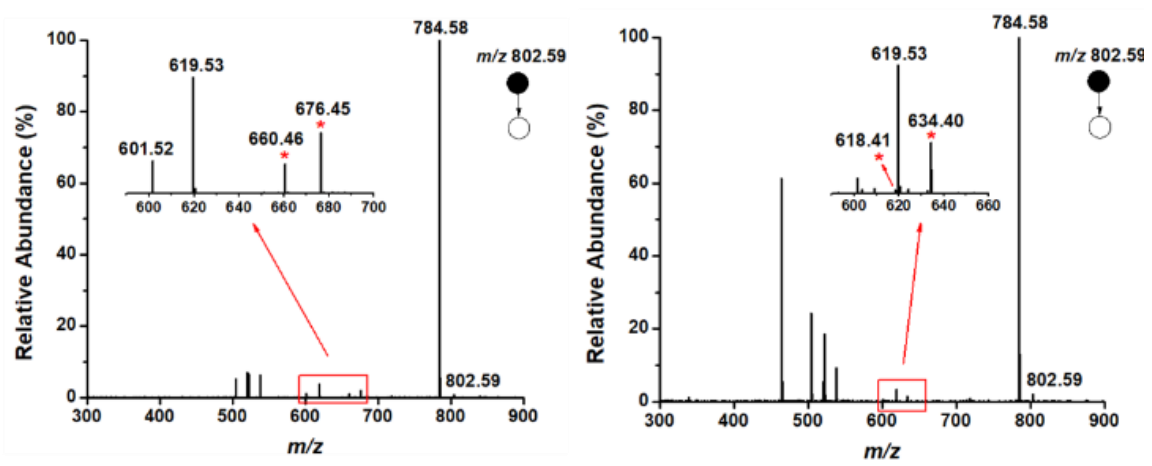

(c) Calibration curve of double-bond isomers

(d) Calibration curve of $s n$ - isomers
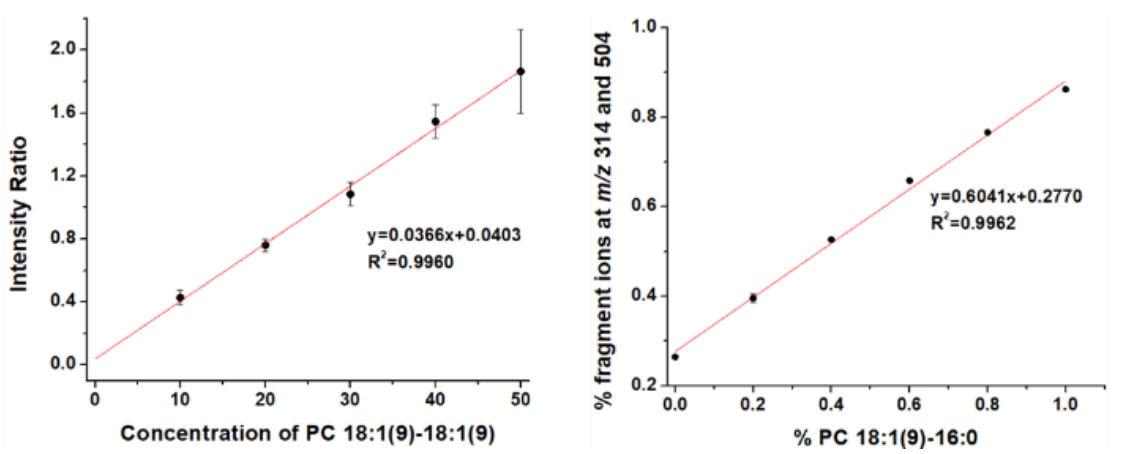

Figure 4. Tandem mass spectra of mono-epoxidized (a) PC 18:1 (9)-18:1 (9) and (b) PC 18:1 (6)-18:1 (6); and the calibration curves of (c) double-bond positional isomers and (d) sn-positional isomers.

\section{Multi-functions of the parameters in EC-MS-based lipid analysis}

EC-MS-based lipid analysis is highly efficient. Many parameters in the design are multifunctional. Voltages that initiate electrospray also control the occurrence of two electrochemical reactions. The cobalt electrode employed 
not only serves as an ESI working electrode, but also provides $\mathrm{Co}^{2+}$ ions via metal electrolysis to form [lipid+Co] ${ }^{2+}$ ions. Hydrochloric acid is the reagent for electro-epoxidation as well as assisting in electrode corrosion to form metal ions.

Metal ions formed in situ by anodic corrosion have many advantages compared to the external addition of salts: i) a metal electrode is typically required in an ESI source, so it can be used readily as a source of metal ions; ii) Release of the metal ions can be controlled by the applied spray voltage; iii) Release of metal ions in a mild and sustained way allows many reactions to occur efficiently; iv) External addition of metal ions often suffers from timeconsuming sample preparation steps and incapacity when unstable metal ions are needed.

\section{Conclusions}

In summary, we have developed a new electrochemical strategy that uses $\mathrm{Co}^{2+}$ formed in situ by voltagecontrolled anodic corrosion of an ESI electrode to achieve the identification of lipid sn-positions due to differentiated fragment pathways of the Co-lipid complexes in CID. Furthermore, we cascade this experiment with interfacial electro-epoxidation to form a novel EC-MS-based workflow for lipid structural characterization. In this workflow, the electro-epoxidation reaction and anodic corrosion of Co electrode are voltage-dependent, therefore the double-bond localization, the sn-position and the lipid base structure can be sequentially determined by tuning ESI voltages. Besides this feature, the on-demand EC-MS workflow for lipid analysis also possesses the following characteristics: (i) low sample consumption; (ii) no requirement for extra instrumentation; (iii) compatibility with the direct infusion lipidomics workflow; (iv) collection of lipid structural information at a high level of specificity using a single sample, and (v) quantitative analysis of lipids isomers. The unique features of this strategy and its simple setup show great potential for its future applications in the lipidomics field.

\section{Corresponding Author}

xyan@tamu.edu

\section{Conflicts of Interest}

There are no conflicts to declare.

\section{Acknowledgments}

We acknowledge the support from Texas A\&M University and the Department of Chemistry.

\section{References}

1. Thiam, A. R.; Dugail, I. J. J. o. c. S., Lipid droplet-membrane contact sites-from protein binding to function. $2019,132(12)$.

2. Phillips, R.; Ursell, T.; Wiggins, P.; Sens, P. J. N., Emerging roles for lipids in shaping membrane-protein function. 2009, 459 (7245), 379385. 
3. Yetukuri, L.; Katajamaa, M.; Medina-Gomez, G.; Seppänen-Laakso, T.; Vidal-Puig, A.; Orešič, M., Bioinformatics strategies for lipidomics analysis: characterization of obesity related hepatic steatosis. BMC Systems Biology 2007, 1 (1), 12.

4. Perry, R. J.; Samuel, V. T.; Petersen, K. F.; Shulman, G. I. J. N., The role of hepatic lipids in hepatic insulin resistance and type 2 diabetes. 2014, 510 (7503), 84-91.

5. Mills, G. B.; Moolenaar, W. H. J. N. R. C., The emerging role of lysophosphatidic acid in cancer. 2003, 3 (8), 582-591.

6. Stegemann, C.; Pechlaner, R.; Willeit, P.; Langley, S. R.; Mangino, M.; Mayr, U.; Menni, C.; Moayyeri, A.; Santer, P.; Rungger, G. J. C., Lipidomics profiling and risk of cardiovascular disease in the prospective population-based Bruneck study. 2014, 129 (18), 1821 -1831.

7. M Jeitner, T.; Voloshyna, I.; B Reiss, A. J. C. m. c., Oxysterol derivatives of cholesterol in neurodegenerative disorders. 2011, 18 (10), 1515-1525.

8. Hinterwirth, H.; Stegemann, C.; Mayr, M. J. C. C. G., Lipidomics: quest for molecular lipid biomarkers in cardiovascular disease. 2014, 7 (6), 941-954.

9. Liu, Q.; Zhang, J. J. N. b., Lipid metabolism in Alzheimer's disease. 2014, 30 (2), 331-345.

10. Musso, G.; Cassader, M.; Paschetta, E.; Gambino, R. J. G., Bioactive lipid species and metabolic pathways in progression and resolution of nonalcoholic steatohepatitis. 2018, 155 (2), 282-302. e8.

11. Jha, P.; McDevitt, M. T.; Halilbasic, E.; Williams, E. G.; Quiros, P. M.; Gariani, K.; Sleiman, M. B.; Gupta, R.; Ulbrich, A.; Jochem, A. J. C. S., Genetic regulation of plasma lipid species and their association with metabolic phenotypes. 2018, 6 (6), 709-721. e6.

12. Quehenberger, O.; Armando, A. M.; Brown, A. H.; Milne, S. B.; Myers, D. S.; Merrill, A. H.; Bandyopadhyay, S.; Jones, K. N.; Kelly, S.; Shaner, R. L.; Sullards, C. M.; Wang, E.; Murphy, R. C.; Barkley, R. M.; Leiker, T. J.; Raetz, C. R. H.; Guan, Z.; Laird, G. M.; Six, D. A.; Russell, D. W.; McDonald, J. G.; Subramaniam, S.; Fahy, E.; Dennis, E. A., Lipidomics reveals a remarkable diversity of lipids in human plasma. $J$ Lipid Res 2010, 51 (11), 3299-3305.

13. Zhao, X.; Zhang, W.; Zhang, D.; Liu, X.; Cao, W.; Chen, Q.; Ouyang, Z.; Xia, Y., A lipidomic workflow capable of resolving sn- and Ca? location isomers of phosphatidylcholines. Chemical Science 2019, 10 (46), 10740-10748.

14. Chatgilialoglu, C.; Ferreri, C.; Melchiorre, M.; Sansone, A.; Torreggiani, A., Lipid Geometrical Isomerism: From Chemistry to Biology and Diagnostics. Chemical Reviews 2014, 114 (1), 255-284.

15. Zhang, S.; Lin, X., Lipid Acyl Chain cis Double Bond Position Modulates Membrane Domain Registration/Anti-Registration. Journal of the American Chemical Society 2019, 141 (40), 15884-15890.

16. Vanni, S.; Riccardi, L.; Palermo, G.; De Vivo, M., Structure and Dynamics of the Acyl Chains in the Membrane Trafficking and Enzymatic Processing of Lipids. Accounts of Chemical Research 2019, 52 (11), 3087-3096.

17. Ellis, S. R.; Hughes, J. R.; Mitchell, T. W.; Blanksby, S. J. J. A., Using ambient ozone for assignment of double bond position in unsaturated lipids. 2012, 137 (5), 1100-1110.

18. Cao, W.; Cheng, S.; Yang, J.; Feng, J.; Zhang, W.; Li, Z.; Chen, Q.; Xia, Y.; Ouyang, Z.; Ma, X., Large-scale lipid analysis with C=C location and sn-position isomer resolving power. Nature Communications 2020, 11 (1), 375.

19. Blanksby, S. J.; Mitchell, T. W., Advances in Mass Spectrometry for Lipidomics. Annual Review of Analytical Chemistry 2010, 3 (1), 433465 .

20. Murphy, R. C.; Fiedler, J.; Hevko, J., Analysis of Nonvolatile Lipids by Mass Spectrometry. Chemical Reviews 2001,101 (2), $479-526$.

21. Rustam, Y. H.; Reid, G. E. J. A. C., Analytical challenges and recent advances in mass spectrometry based lipidomics. 2018, 90 (1), $374-397$.

22. Lam, C.; Jie, M. L. K. J. C.; Lipids, P. O., Fatty acids, part 5: A study of the oxymercuration-demercuration reaction of some C11-unsaturated fatty esters and methyl octadec-cis-10-en-5-ynoate. 1976, 16 (3), 181-194.

23. Minnikin, D. J. C.; Lipids, P. O., Location of double bonds and cyclopropane rings in fatty acids by mass spectrometry. 1978, 21 (4), $313-$ 347.

24. Francis, G. W. J. C.; Lipids, P. o., Alkylthiolation for the determination of double-bond position in unsaturated fatty acid esters. 1981,29 (4), 369-374.

25. Buser, H. R.; Arn, H.; Guerin, P.; Rauscher, S. J. A. C., Determination of double bond position in mono-unsaturated acetates by mass spectrometry of dimethyl disulfide adducts. 1983, 55 (6), 818-822.

26. Leonhardt, B.; DeVilbiss, E. J. J. o. C. A., Separation and double-bond determination on nanogram quantities of aliphatic monounsaturated alcohols, aldehydes and carboxylic acid methyl esters. 1985, 322, 484-490.

27. Kwon, Y.; Lee, S.; Oh, D. C.; Kim, S. J. A. C., Simple Determination of Double-Bond Positions in Long-Chain Olefins by Cross-Metathesis. 2011, $123(36), 8425-8428$.

28. Harrison, K. A.; Murphy, R. C. J. A. c., Direct mass spectrometric analysis of ozonides: Application to unsaturated glycerophosphocholine lipids. 1996, 68 (18), 3224-3230.

29. Thomas, M. C.; Mitchell, T. W.; Blanksby, S. J., Ozonolysis of Phospholipid Double Bonds during Electrospray lonization: A New Tool for Structure Determination. Journal of the American Chemical Society 2006, 128 (1), 58-59.

30. Zhang, J. I.; Tao, W. A.; Cooks, R. G. J. A. C., Facile determination of double bond position in unsaturated fatty acids and esters by low temperature plasma ionization mass spectrometry. 2011, 83 (12), 4738-4744.

31. Ma, X.; Xia, Y., Pinpointing Double Bonds in Lipids by Paternò-Büchi Reactions and Mass Spectrometry. Angewandte Chemie International Edition 2014, 53 (10), 2592-2596.

32. Ma, X.; Chong, L.; Tian, R.; Shi, R.; Hu, T. Y.; Ouyang, Z.; Xia, Y., Identification and quantitation of lipid C=C location isomers: A shotgun lipidomics approach enabled by photochemical reaction. Proceedings of the National Academy of Sciences 2016, 113 (10), 2573.

33. Ma, X.; Zhao, X.; Li, J.; Zhang, W.; Cheng, J.-X.; Ouyang, Z.; Xia, Y., Photochemical Tagging for Quantitation of Unsaturated Fatty Acids by Mass Spectrometry. Analytical Chemistry 2016, 88 (18), 8931-8935.

34. Murphy, R. C.; Okuno, T.; Johnson, C. A.; Barkley, R. M. J. A. C., Determination of double bond positions in polyunsaturated fatty acids using the photochemical Paterno-Buchi reaction with acetone and tandem mass spectrometry. 2017, 89 (16), $8545-8553$. 
35. Cao, W.; Ma, X.; Li, Z.; Zhou, X.; Ouyang, Z., Locating Carbon-Carbon Double Bonds in Unsaturated Phospholipids by Epoxidation Reaction and Tandem Mass Spectrometry. Analytical Chemistry 2018, 90 (17), 10286-10292.

36. Zhao, X.; Zhao, Y.; Zhang, L.; Ma, X.; Zhang, S.; Zhang, X., Rapid Analysis of Unsaturated Fatty Acids on Paper-Based Analytical Devices via Online Epoxidation and Ambient Mass Spectrometry. Analytical Chemistry 2018, 90 (3), 2070-2078.

37. Wan, L.; Gong, G.; Liang, H.; Huang, G., In situ analysis of unsaturated fatty acids in human serum by negative-ion paper spray mass spectrometry. Analytica Chimica Acta 2019, 1075, 120-127.

38. Feng, Y.; Chen, B.; Yu, Q.; Li, L., Identification of Double Bond Position Isomers in Unsaturated Lipids by m-CPBA Epoxidation and Mass Spectrometry Fragmentation. Analytical Chemistry 2019, 91 (3), 1791-1795.

39. Kuo, T.-H.; Chung, H.-H.; Chang, H.-Y.; Lin, C.-W.; Wang, M.-Y.; Shen, T.-L.; Hsu, C.-C., Deep Lipidomics and Molecular Imaging of Unsaturated Lipid Isomers: A Universal Strategy Initiated by mCPBA Epoxidation. Analytical Chemistry 2019, 91 (18), 11905-11915.

40. Klein, D. R.; Brodbelt, J. S. J. A. C., Structural characterization of phosphatidylcholines using $193 \mathrm{~nm}$ ultraviolet photodissociation mass spectrometry. 2017, 89 (3), 1516-1522.

41. Poad, B. L.; Green, M. R.; Kirk, J. M.; Tomczyk, N.; Mitchell, T. W.; Blanksby, S. J. J. A. C., High-pressure ozone-induced dissociation for lipid structure elucidation on fast chromatographic timescales. 2017, 89 (7), 4223-4229.

42. Baba, T.; Campbell, J. L.; Le Blanc, J. Y.; Baker, P. R. J. J. o. I. r., Structural identification of triacylglycerol isomers using electron impact excitation of ions from organics (EIEIO). 2016, 57 (11), 2015-2027.

43. Williams, P. E.; Klein, D. R.; Greer, S. M.; Brodbelt, J. S. J. J. o. t. A. C. S., Pinpointing double bond and sn-positions in glycerophospholipids via hybrid $193 \mathrm{~nm}$ ultraviolet photodissociation (UVPD) mass spectrometry. 2017, 139 (44), 15681-15690.

44. Becher, S.; Esch, P.; Heiles, S., Relative Quantification of Phosphatidylcholine sn-Isomers Using Positive Doubly Charged Lipid-Metal lon Complexes. Analytical Chemistry 2018, 90 (19), 11486-11494.

45. Paine, M. R. L.; Poad, B. L. J.; Eijkel, G. B.; Marshall, D. L.; Blanksby, S. J.; Heeren, R. M. A.; Ellis, S. R., Mass Spectrometry Imaging with Isomeric Resolution Enabled by Ozone-Induced Dissociation. Angewandte Chemie International Edition 2018, 57 (33), $10530-10534$.

46. Baba, T.; Campbell, J. L.; Le Blanc, J. C. Y.; Baker, P. R. S., Distinguishing Cis and Trans Isomers in Intact Complex Lipids Using Electron Impact Excitation of Ions from Organics Mass Spectrometry. Analytical Chemistry 2017, 89 (14), 7307-7315.

47. Tang, S.; Cheng, H.; Yan, X., On-Demand Electrochemical Epoxidation in Nano-Electrospray lonization Mass Spectrometry to Locate Carbon-Carbon Double Bonds. Angewandte Chemie International Edition 2020, 59 (1), 209-214. 